\title{
Statistical Similarity Based Change Detection for Multitemporal Remote Sensing Images
}

\author{
Mumu Aktar, Md. Al Mamun, and Md. Ali Hossain \\ Computer Science \& Engineering Department, Rajshahi University of Engineering \& Technology, Rajshahi 6204, Bangladesh \\ Correspondence should be addressed to Mumu Aktar; mumu.ruet@gmail.com
}

Received 11 March 2017; Revised 23 May 2017; Accepted 14 June 2017; Published 24 July 2017

Academic Editor: Huan Xie

Copyright ( 2017 Mumu Aktar et al. This is an open access article distributed under the Creative Commons Attribution License, which permits unrestricted use, distribution, and reproduction in any medium, provided the original work is properly cited.

\begin{abstract}
Change detection (CD) of any surface using multitemporal remote sensing images is an important research topic since up-to-date information about earth surface is of great value. Abrupt changes are occurring in different earth surfaces due to natural disasters or man-made activities which cause damage to that place. Therefore, it is necessary to observe the changes for taking necessary steps to recover the subsequent damage. This paper is concerned with this issue and analyzes statistical similarity measure to perform $\mathrm{CD}$ using remote sensing images of the same scene taken at two different dates. A variation of normalized mutual information (NMI) as a similarity measure has been developed here using sliding window of different sizes. In sliding window approach, pixels' local neighborhood plays a significant role in computing the similarity compared to the whole image. Thus the insignificant global characteristics containing noise and sparse samples can be avoided when evaluating the probability density function. Therefore, NMI with different window sizes is proposed here to identify changes using multitemporal data. Experiments have been carried out using two separate multitemporal remote sensing images captured one year apart and one month apart, respectively. Experimental analysis reveals that the proposed technique can detect up to $97.71 \%$ of changes which outperforms the traditional approaches.
\end{abstract}

\section{Introduction}

Change detection $(\mathrm{CD})$ plays an important role in earth observation since a lot of changes occurring on different areas of earth surface cause severe damage to that area. So, it is very important to identify changes occurring on that area to take steps for subsequent recovery from damage. Most of the damage is caused due to natural disasters like flooding, rainfall, and droughts and also by man-made activities and thus it is necessary to observe the areas on regular intervals for detecting that change. Multitemporal remote sensing images taken at different times provide the periodic repeated look of an area which is very necessary for regular observation of earth surface.

The spatial, radiometric, spectral, and temporal resolution of an image has been improved due to recent advances in satellite imaging that has made earth observations much easier [1]. Multispectral remote sensing image is a valuable source of satellite data providing necessary information by each band, that is why in this research two separate multispectral datasets of two different dates have been chosen to identify changes. CD is applied to different tasks including land cover change detection [2,3], building change detection $[4,5]$, vegetation change detection [6], wetland monitoring [7], and so on. Generally, CD detection can be categorized in two ways: binary change detection and multiclass change detection. Binary change detection focuses on change versus no change whereas multiclass change detection uses some supervised or unsupervised approaches for classifying multiple classes and further identifies changes of each class separately. This paper is concerned with binary change detection. A number of different techniques are used for binary change detection such as image differencing [8, 9], log-ratio operator [10], Kullback Leibler divergence [11], regression analysis [12], vegetation index differencing $[13,14]$, and principal component analysis $[1,15]$.

Ground truth data is needed for supervised approach being used for change detection. When this ground truth or prior knowledge is unavailable then threshold selection is very important to take decision about change/no change. An 
adaptive threshold can be used for generating a binary change detection map. Change detection of multisensor images at pixel level is verified using manual threshold which faced false alarms because only the mean value is considered for threshold selection [3]. Here in this research a difference image is generated using image differencing method which requires a threshold to take decision about changes. OTSU method for threshold selection $[16,17]$ has been used here since it maximizes variance between changed and unchanged pixels for generating a binary change detection map.

Since threshold used in image differencing method depends on radiometric differences of pixel values, singly it is not always enough to determine changes between two times' images since they can provide false detection due to registration errors, that is why a new approach is proposed here based on statistical similarity to determine the percentage of changes along with image differencing. In [3] a number of similarity measure techniques such as distance to independence, normalized standard deviation or Woods criterion, correlation ratio, mutual information (MI), and cluster reward algorithm (CRA) have been applied on land cover change detection for multisensor remotely sensed images and the performances have been compared in case of two separate fixed window sizes. Mean value of similarity image has been used there as a threshold for decision about change/no change of any pixel. Although result of the statistical measures has been normalized, optimal dimension of the estimated windows has not been investigated. Land cover change detection has been performed in [2] considering sliding window by transforming spectral values into local spectrum trend using curve fitting and raster encoding techniques. This approach is time consuming and even difficult when calculating the local trend spectrum for different window sizes. Multisensor remote sensing image change detection using mutual information, maximal information coefficient, and distance correlation measure based similarity measures has been developed in [18] using sliding window approach with fixed window size of $21 \times 21$. This approach used manual threshold for interpreting change and no change regions and generated false positives. Selection of threshold is a difficult task there as it is justified manually.

Among a number of similarity measures, MI has been chosen here for change detection purpose because of its many advantages. MI is a nonparametric approach that does not require any assumption about the shape of the distribution of input variables and able to measure both the linear and nonlinear relationships among input variables [19]. A lot of papers considered MI as a similarity measure for change detection task including multivariate statistical model [20] which concentrated on homogeneous and heterogeneous sensors, SAR image change detection [21] which considered different kind of changes, canonical information analysis [22] for image change detection, multicontextual mutual information data as an improved form of image spatial mutual information [23] for SAR image change detection, and temporal behavior of multichannel scene characterization for change detection [24].

But the value of MI is affected by the entropies of the input variables and the calculated MI without being normalized cannot measure similarity effectively, so the MI measure can be improved by normalizing it to a specified range such as $[0,1][19]$, that is why MI being normalized (NMI) has been analyzed here in this research work as a similarity measure to detect changes. But when just traditional NMI is applied on the whole image then a pixels' marginal and joint probability density functions (PDFs) are affected with the globally spread information that can be noisy and has less significance on them. But if sliding window with varying window sizes is considered then pixels' local neighborhood is used for calculating PDF which is the most significant one. The window which is too small or too large affects the CD results [2]. So different window sizes have been considered here in this research to find out which one is more efficient for better change detection result. Thus to significantly perform change detection on multitemporal remote sensing images sliding window based normalized mutual information (SWBNMI) with varying window sizes has been considered here to statistically detect changes using 1-Imagesimilarity $(\mathbf{X}, \mathbf{Y})$ concept. Experimental analysis has been performed using remotely sensed multispectral imagery of Canberra region of Australia with multitemporal datasets of two separate dates.

The rest of the paper is organized as follows. Section 2 provides a clear concept of proposed change detection method. The proposed methodology for multispectral images' change detection is presented in Section 3. In Section 4 experimental analysis and performance evaluation of the proposed method have been performed. Finally, a conclusion is given in Section 5.

\section{Proposed Change Detection Method}

2.1. OTSU Threshold Selection Method. Threshold selection is a key concept used in unsupervised approaches for generating a binary change detection map. OTSU's method is a popular approach which maximizes variance between changed and unchanged pixels to get an optimal threshold [17]. The OTSU algorithm assumes the image containing two classes of pixels as foreground pixels and background pixels; it then calculates the optimum threshold separating the two classes to minimize their combined spread (intraclass variance), $V 1$, or equivalently (because the sum of pairwise squared distances is constant), to maximize their interclass variance, $V$ [16]. The optimal threshold, $V$, can be obtained by maximizing the following criteria with respect to $V 1$ [17]:

$$
\eta=\theta_{0} \times \theta_{1} \times\left(\mu_{0}-\mu_{1}\right)^{2}
$$

where $\theta_{0}$ and $\mu_{0}$ are the count and mean value of elements in difference image, $D$ which are less than $V 1$, and $\theta_{1}$ and $\mu_{1}$ are the count and mean value of elements in difference image, $D$ which are no less than $V 1$.

Finally the decision about change/no change can be defined as [17]

$$
\text { decision function, } d(k)= \begin{cases}\text { change } & \text { if } d(k) \geq v \\ \text { no change } & \text { if } d(k)<v\end{cases}
$$




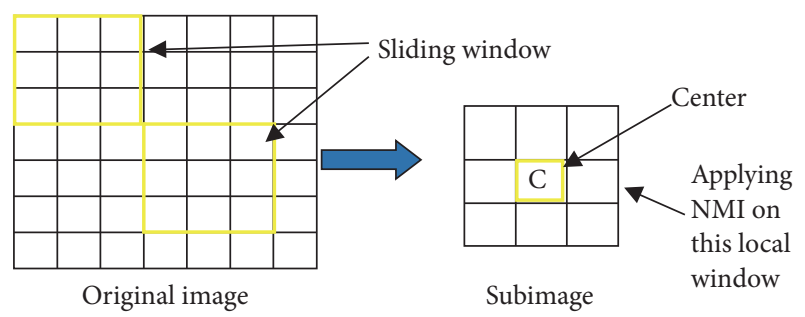

FIGURE 1: Basic concept of sliding window approach.

2.2. NMI as a Statistical Similarity Measure. MI of two discrete random variables $\mathbf{X}$ and $\mathbf{Y}$ can be defined as [19] using Shannon entropy theorem [25]:

$$
I(\mathbf{X}, \mathbf{Y})=\sum_{x \in \mathbf{X}} \sum_{y \in \mathbf{Y}} p(x, y) \log \left(\frac{p(x, y)}{p(x) p(y)}\right),
$$

where $p(x, y), p(x)$, and $p(y)$ are the joint and marginal probability distribution functions of $\mathbf{X}$ and $\mathbf{Y}$, respectively. MI between these two variables is zero if they are independent of each other and will be higher if they are similar to some degree. If the MI value is normalized to the range between 0 1 then the effect of input variables' entropy can be removed and thus MI can be used as an improved similarity measure. Therefore, NMI has been applied here to identify percentage of changes between two images $\mathbf{X}$ and $\mathbf{Y}$ in which one is the reference and the other is the target image. The NMI is defined as [19]

$$
\dot{I}(\mathbf{X}, \mathbf{Y})=\frac{I(\mathbf{X}, \mathbf{Y})}{\sqrt{H(\mathbf{X})} \sqrt{H(\mathbf{Y})}},
$$

where $H(\mathbf{X})$ is the entropy of the variable $\mathbf{X}$ which represents the amount of information held in $\mathbf{X}$ and the same representation in case of $H(\mathbf{Y})$.

2.3. SWB-NMI as a Statistical Similarity Measure. Figure 1 is an example showing the basic concept of how sliding window can be moved over an image to apply NMI on each local window. An efficient way for remote sensing image change detection is to analyze the image's local geographical layout for its similarity measure. For this purpose the sliding window approach can be considered where a change indicator is applied separately on each window. Thus the statistical properties such as joint and marginal probabilities of the pixels can be calculated from the local neighborhood information which are most significant ones. Different window sizes are considered here to achieve the relevant information. Window sizes such as $3 \times 3,5 \times 5,9 \times 9,13 \times 13,15 \times 15,23 \times 23$, and $27 \times 27$ are used instead of full image to avoid the global statistics of pixels which may contain irrelevant information. Thus SWBNMI has been chosen in this research with an expectation of detecting changes locally and the initial results are promising. Since a large window results noise and small window do not contain enough spatial contextual information [2], varying sized windows have been applied to the experiment here to find an optimal window size and the performances have been compared with traditional approaches stated in $[2,3]$.

\section{Proposed Methodology}

The objective of this research is to identify changes between the same scenes taken at different times to find out the places of any area which may be damaged due to unintended occurrences. For this work two separate multitemporal datasets of two different dates have been chosen. Landsat 7 ETM+ multispectral images have been chosen for this experiment which consists of eight spectral bands with a spatial resolution of 30 meters for bands 1 to 7 and 15 meters for the panchromatic band 8. Among them six bands from 1 to 5 and 7 (band 6 collects both high and low gain for all scenes whereas all other bands collect one of two gains and band 8 has different resolution compared to the others and that is why bands 6 and 8 are ignored here) have been chosen here.

In 1st multispectral dataset, two Landsat ETM+ images of $256 * 256$ pixels have been taken one year apart of 2000 and 2001 from Canberra region of Australia.

The 2nd dataset is from another part of Canberra region captured around one month apart at 20.11.2016 and 22.12.2016. These images consist of $512 * 512$ pixels. The overall working methodology is stated in Figure 2 as a flowchart.

Let us consider individually the multispectral images of 2000 and 20.11.2016 as the reference image, $\mathbf{X}$, and images of 2001 as well as 22.12 .2016 as the target image Y. Simply the difference between these multitemporal scenes is obtained by the following [26]:

$$
\text { Difference Image, } \mathbf{I}_{\mathbf{D}}=\mathbf{X}-\mathbf{Y}
$$

Equation (5) subtracts each pixel of target images from each pixel of the reference ones. Since pixel-to-pixel differencing is performed here, so it is very necessary for the reference and target images to be coregistered. Using the concept of (1) a binary change map has been generated by automatic threshold selected using OTSU algorithm. The image differencing has been performed here band by band and the binary change detection map is shown only for band 1 for both multitemporal datasets.

The reference and the target images and the corresponding binary change maps based on the selected threshold value for both datasets are shown in Figure 3.

It can be seen from Figure 3(j) that the binary change map of 2 nd dataset contains very little amount of changes, since it is the multitemporal data of one month interval. The red points are defined as changed areas. In one month perhaps some buildings, parks can be changed which can be some pixels in change map image. Since they are very small points, so for better visual interpretation the points are modified as large. It is found in Figures 3(d) and 3(i) that the tail portion of each of the histograms represent the change portion as it is expected that intensity differences due to land cover change reside at the tails of the difference distribution of any image [27].

From this binary change map of Figure 3(e), it can be obtained that around $35 \%$ area in the reference image has been changed within one year which has been calculated by subtracting the total number of changed pixels from the total 


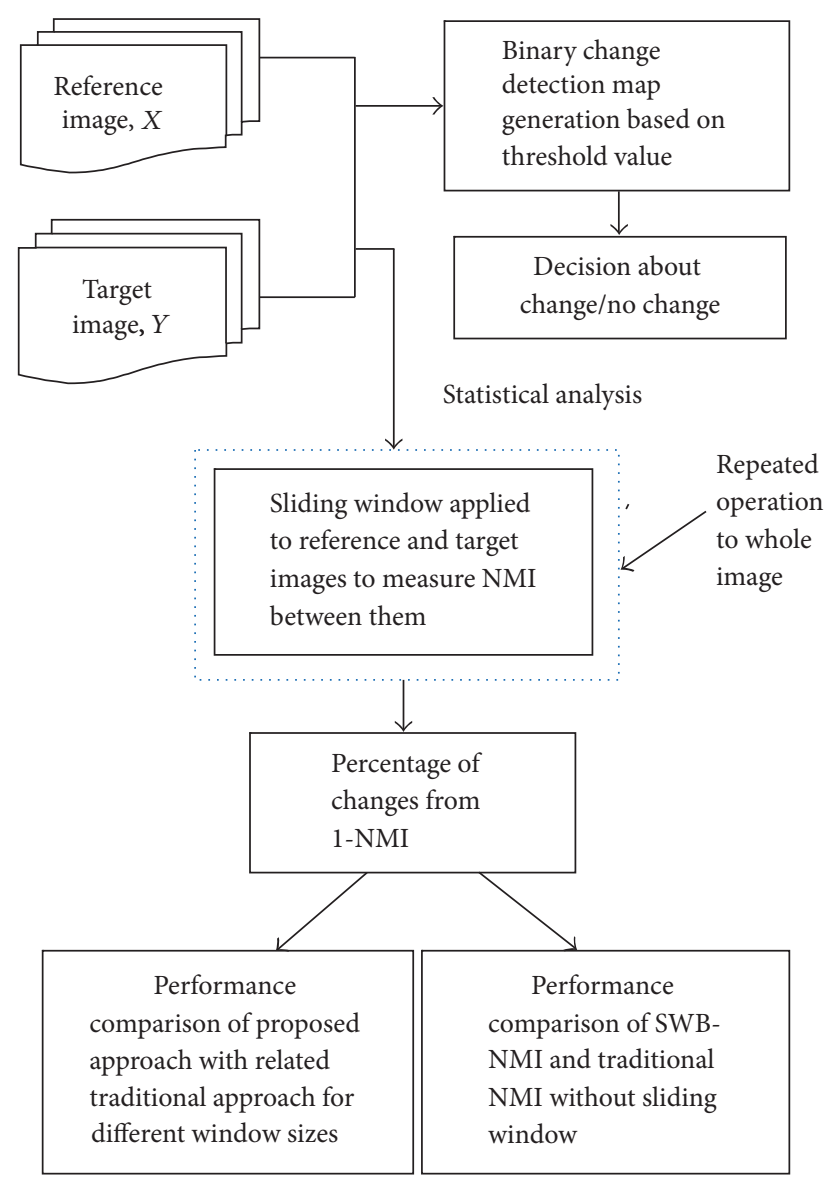

Figure 2: Methodology of proposed change detection system.

number of pixels contained on the reference image, dividing the resulting value by the total number of reference image's pixels, and then taking the percentage value of the result obtained. The same method has been applied for 2 nd dataset and here around $4 \%$ of area has been changed which can be observed from Figure 3(j).

After obtaining the binary change map, statistical similarity has been measured between target and reference images for calculating percentage of changes in both datasets. In both cases, same window for both the reference and target ones is selected for applying NMI on them to measure similarity and find $\mathrm{CD}$ by 1 -Imagesimilarity $(\mathbf{X}, \mathbf{Y})$. The sliding window has been iteratively applied to the whole image area starting at location $(1,1)$. And the process has been repeated for different window sizes and the performances have been compared with [2]. Finally the performance of CD of the proposed SWBNMI method has been compared with the traditional MI in [3].

\section{Experimental Analysis and Performance Evaluation}

Change detection operation has been performed using two separate multitemporal multispectral datasets of separate portions of Canberra region, Australia. Percentage of correct change detection from these multitemporal images has been calculated as (6):

Percentage of correct change detection

$$
\begin{aligned}
& =1 \\
& \quad-\operatorname{abs}\left(\frac{\text { original change }- \text { experimental change }}{\text { original change }}\right) \\
& \quad \times 100 \% .
\end{aligned}
$$

It has been obtained from the binary change maps generated by considering threshold values is that the original changes which occurred in the reference images are around $35 \%$ and $4 \%$ for two datasets, respectively. Therefore, using these values and the percentage of experimental changes, Tables 1-5 show the experimental results on the amount of changes in percentage for different window sizes using the proposed SWB-NMI technique for both datasets. The results of both datasets for different window sizes have been compared with [2].

From Table 1 it can be seen that up to $97.71 \%$ of changes can be correctly detected using SWB-NMI approach for window size of 17 and it varies a little for window size of 15 . Figure 4 represents the performance of varying window sizes 


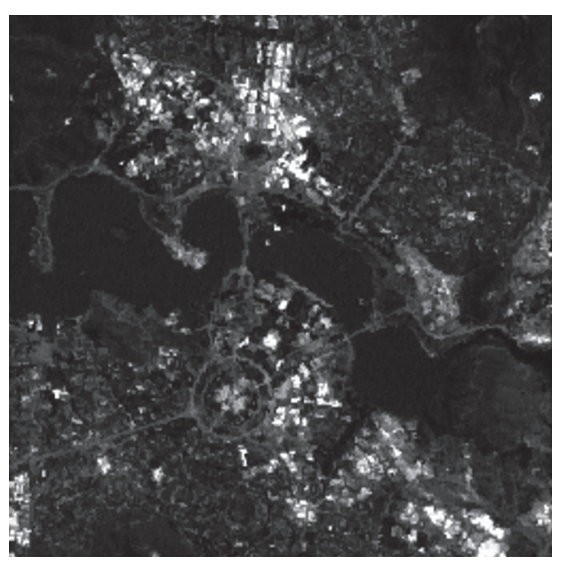

(a)

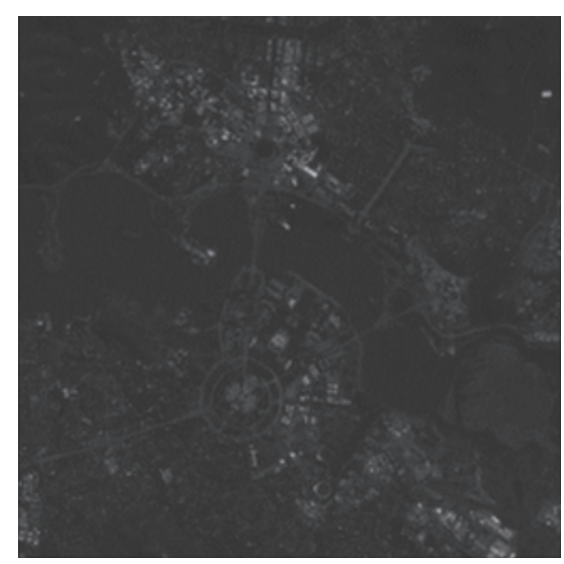

(b)

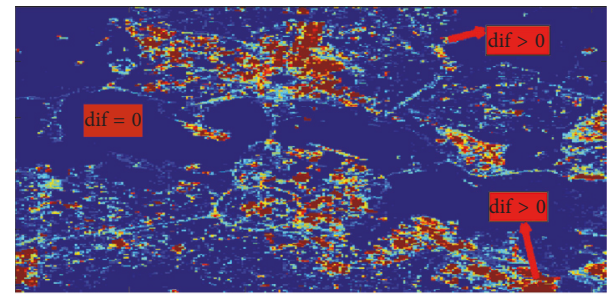

(c)

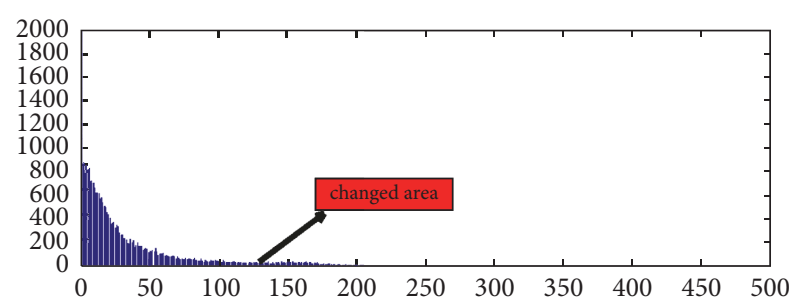

(d)
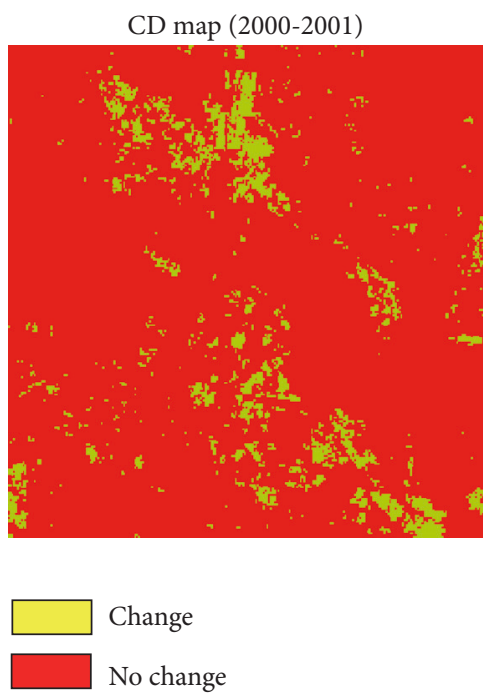

(e)

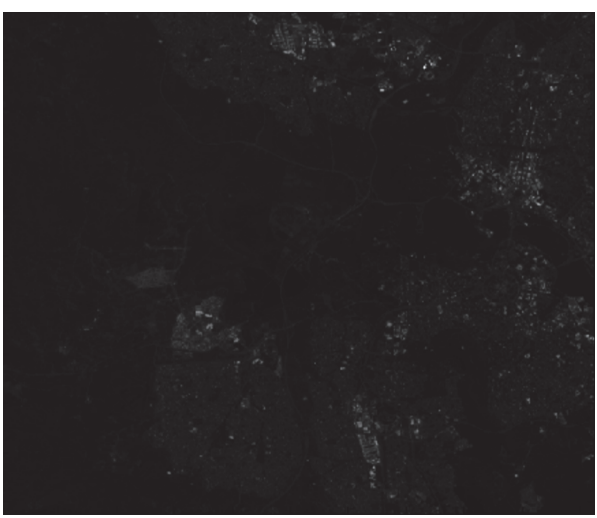

(f)

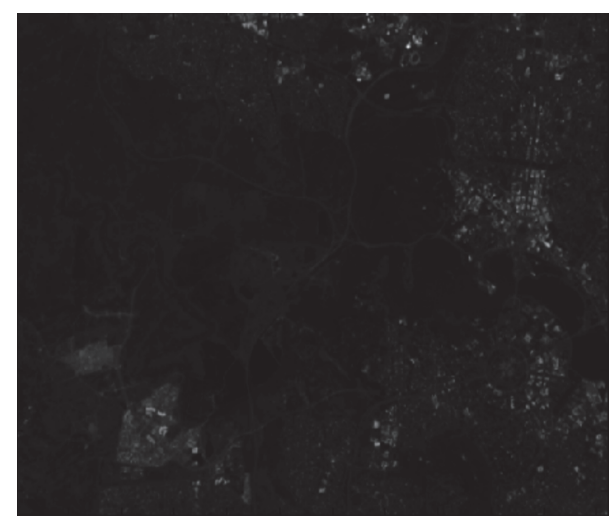

(g)

Figure 3: Continued. 


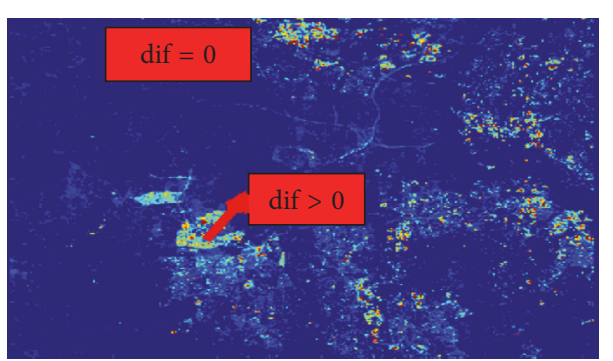

(h)

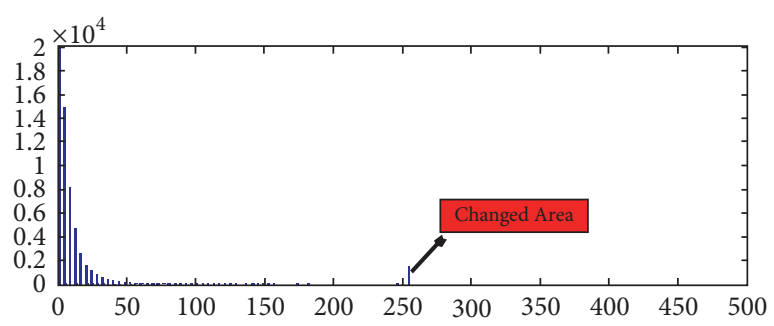

(i)

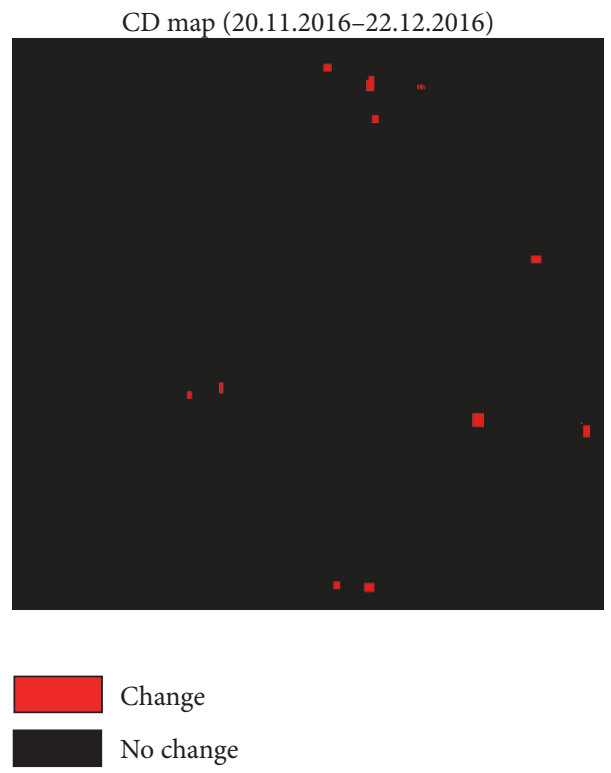

(j)

FIGURE 3: Experimental images and change map by OTSU threshold selection. (a) Reference image: 2000. (b) Target image: 2001. (c) Difference image. (d) Histogram of difference image. (e) Corresponding binary change map. (f) Reference image: 20.11.2016. (g) Target image: 22.12.2016. (h) Difference image. (i) Histogram of difference image. (j) Corresponding change map.

TABLE 1: Percentage of changes using SWB-NMI for different window sizes (1st dataset).

\begin{tabular}{lcccc}
\hline $\begin{array}{l}\text { Window } \\
\text { size }\end{array}$ & $\begin{array}{c}\text { \% of } \\
\text { similarities }\end{array}$ & $\begin{array}{c}\text { \% of } \\
\text { changes }\end{array}$ & $\begin{array}{c}\text { \% of correct } \\
\text { change detection }\end{array}$ & $\begin{array}{c}\text { \% of wrong } \\
\text { change detection }\end{array}$ \\
\hline 5 & 70.0 & 30.0 & 85.71 & 14.28 \\
9 & 68.0 & 32.0 & 91.43 & 8.57 \\
13 & 63.5 & 36.5 & 95.71 & 4.29 \\
15 & 64.0 & 36.0 & 97.14 & 2.86 \\
17 & $\mathbf{6 4 . 2}$ & 35.8 & 97.71 & 2.29 \\
23 & 61.0 & 39.0 & 88.57 & 11.43 \\
27 & 59.5 & 40.5 & 84.29 & 15.71 \\
\hline
\end{tabular}

in case of 1st dataset using SWB-NMI for change detection application.

Table 2 compares the performance of proposed approach with the related model given in [2] where $3 \times 3,5 \times 5,7 \times 7$, and $9 \times 9$ window sizes were considered.

Although performance has been compared between these two methods for only $5 \times 5$ and $9 \times 9$ window sizes, from Table 1 it is seen that window size $17 \times 17$ shows the best performance.
TABLE 2: Performance comparison in percentage between SWBNMI and approach in [2] with different window sizes for 1st dataset.

\begin{tabular}{lcc}
\hline Method & Window size, $5 \times 5$ & Window size, $9 \times 9$ \\
\hline SWB-NMI & 85.71 & 91.43 \\
Approach in [2] & 84.3 & 85.3 \\
\hline
\end{tabular}

Experiment has been performed for 2nd dataset using the same window sizes as used in [2] for better performance comparison.

In Table 3 the proposed approach has obtained up to $95 \%$ of correct changes with window size of 11 for 2 nd dataset.

Now the performance of the proposed approach has been compared with the approach of [2] in Table 4.

Here it is observed that the proposed approach has shown better performance than the related one in [2]. It can also be seen that although the proposed approach has shown highest performance for window size 17 in dataset-1, it has shown highest performance in case of dataset- 2 for window size 11. It can be concluded from this scenario that performance depends partially on the dataset also. Also the result has varied a lot when the proposed SWB_NMI approach being 


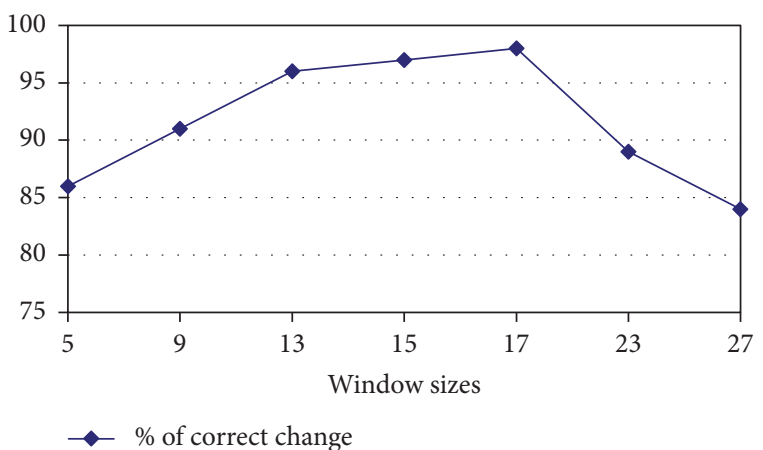

FIGURE 4: Relationship between window sizes and (\%) of correct changes.

TABle 3: Percentage of changes using SWB-NMI for different window sizes (2nd dataset).

\begin{tabular}{lcccc}
\hline $\begin{array}{l}\text { Window } \\
\text { size }\end{array}$ & $\begin{array}{c}\text { \% of } \\
\text { similarities }\end{array}$ & $\begin{array}{c}\text { \% of } \\
\text { changes }\end{array}$ & $\begin{array}{c}\text { \% of correct } \\
\text { change detection }\end{array}$ & $\begin{array}{c}\text { \% of wrong } \\
\text { change detection }\end{array}$ \\
\hline 3 & 96.7 & 3.3 & 82.5 & 17.5 \\
5 & 96.6 & 3.4 & 85 & 15 \\
7 & 96.5 & 3.5 & 87.5 & 12.5 \\
9 & 96.3 & 3.7 & 92.5 & 7.5 \\
11 & 96.2 & 3.8 & 95 & 5 \\
13 & 95.6 & 4.4 & 90 & 10 \\
15 & 95.5 & 4.49 & 87.75 & 12.25 \\
\hline
\end{tabular}

TABLE 4: Performance comparison in percentage between SWBNMI and approach in [2] with different window sizes for 2 nd dataset.

\begin{tabular}{lcccc}
\hline Method & $\begin{array}{c}\text { Window } \\
\text { size, } 3 \times 3\end{array}$ & $\begin{array}{c}\text { Window } \\
\text { size, } 5 \times 5\end{array}$ & $\begin{array}{c}\text { Window } \\
\text { size, } 7 \times 7\end{array}$ & $\begin{array}{c}\text { Window } \\
\text { size, } 9 \times 9\end{array}$ \\
\hline SWB-NMI & 82.5 & 85 & 87.5 & 92.5 \\
Approach in [2] & 82.1 & 84.3 & 84.8 & 85.3 \\
\hline
\end{tabular}

TABLE 5: Change detection result based on SWB-NMI and traditional MI used in [3].

\begin{tabular}{lccc}
\hline Methods & $\begin{array}{r}\text { SWB-NMI } \\
\text { dataset-1 }\end{array}$ & $\begin{array}{r}\text { SWB-NMI } \\
\text { dataset-2 }\end{array}$ & MI in [3] \\
\hline $\begin{array}{l}\text { \% of correct change } \\
\text { detection }\end{array}$ & 97.71 & 95 & 86.1 \\
\hline
\end{tabular}

used as a similarity measure has been compared to the related local trend similarity approach and the proposed method has shown better result.

Figure 5 represents the performance of varying window sizes in case of 2 nd dataset using SWB-NMI for change detection application.

It can be noticed from Figures 4 and 5 that the smaller as well as larger window size does not mean to have better detection ability. For instance, in case of dataset- 1 the lowest value of $84.29 \%$ of correct changes is found for the largest $27 \times 27$ window size and the same case for the smallest $5 \times 5$ window with $85.71 \%$ of correct changes. The same case happens for dataset- 2 with window sizes $3 \times 3$ and $15 \times 15$.

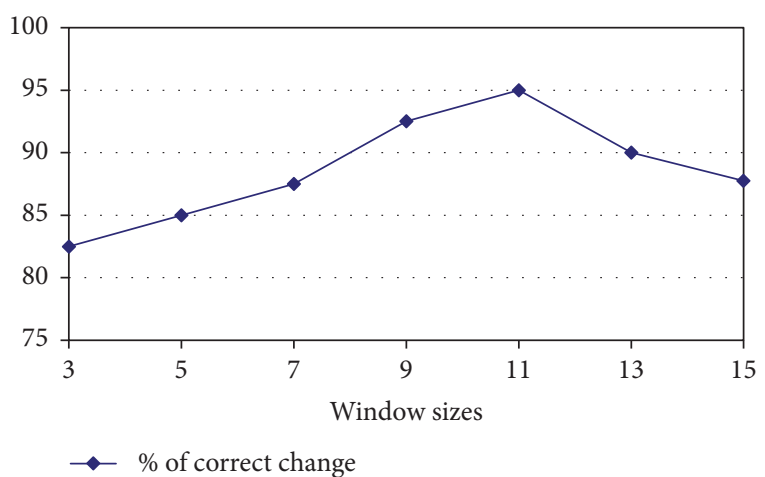

FIGURE 5: Relationship between window sizes and (\%) of correct changes.

It can be observed from the above results that the $\mathrm{CD}$ result is affected with varying window sizes and both the smallest and the largest ones lead to the decrease in detection performance and detect the unchanged ones as the changed and vice versa. It is because when a local window is too small then it will not contain enough spatial contextual information and when it is too large then it will contain a lot of noisy as well as irrelevant information. Thus it can be concluded that window size should be an optimal one which is not very large not very small. 17 and 11 are the better and optimal ones for this experiment although it depends on dataset.

Finally, Table 5 shows the performance of proposed SWBNMI being compared with traditional MI in [3].

From Table 5 it can be observed that the SWB-NMI (for $17 \times 17$ window size) can detect $97.71 \%$ of changes in case of dataset-1. Although the percentage is less in case of dataset-2, both outperforms the related method used in [3].

\section{Conclusion}

An efficient change detection approach has been proposed here in this research to identify changes of any geographical area after some natural disasters or artificial changes made by human being. At first a binary change map has been generated using OTSU threshold selection method to show the change/no change area and further statistical similarity has been measured between reference and target images to find percentage of changes. This approach results better change detection by statistical analysis of similarity measure and by considering the local neighborhood characteristics which are insignificant most of the time rather than the global ones. An important finding is also from this research that window size is an important factor when using local window for statistical analysis of similarity. Thus the SWBNMI approach outperforms the traditional MI method in case of change detection. Image differencing is performed here on pixel-by-pixel basis for generating change detection map, which may often face the registration error and the final performance may be affected greatly. So further interest is to investigate some developed methods which do not directly depend on radiometric values. This research focuses on binary change detection whereas the multiclass change 
detection is more challenging task which is also a future research interest.

\section{Conflicts of Interest}

The authors declare that there are no conflicts of interest regarding the publication of this paper.

\section{References}

[1] M. Al Mamun, M. N. I. Mondal, and B. Ahmed, "Change detection-aided single linear prediction of multi-temporal satellite images," in Proceedings of the 17th International Conference on Computer and Information Technology, ICCIT '14, pp. 332-335, IEEE, Dhaka, Bangladesh, December 2014.

[2] P. Zhang, Z. Lv, and W. Shi, "Local spectrum-trend similarity approach for detecting land-cover change by using SPOT-5 satellite images," IEEE Geoscience and Remote Sensing Letters, vol. 11, no. 4, pp. 738-742, 2014.

[3] V. Alberga, "Similarity measures of remotely sensed multisensor images for change detection applications," Remote Sensing, vol. 1, no. 3, pp. 122-143, 2009.

[4] J. Tian, S. Cui, and P. Reinartz, "Building change detection based on satellite stereo imagery and digital surface models," IEEE Transactions on Geoscience and Remote Sensing, vol. 52, no. 1, pp. 406-417, 2014.

[5] D.-J. Kim, S. Hensley, S.-H. Yun, and M. Neumann, "Detection of durable and permanent changes in urban areas using multitemporal polarimetric UAVSAR data," IEEE Geoscience and Remote Sensing Letters, vol. 13, no. 2, pp. 267-271, 2016.

[6] S. Liu, L. Bruzzone, F. Bovolo, and P. Du, "Unsupervised hierarchical spectral analysis for change detection in hyperspectral images," in Proceedings of the 2012 4th Workshop on Hyperspectral Image and Signal Processing, WHISPERS '12, IEEE, Shanghai, China, June 2012.

[7] L. Moser, S. Voigt, E. Schoepfer, and S. Palmer, "Multitemporal wetland monitoring in sub-Saharan West-Africa using medium resolution optical satellite data," IEEE Journal of Selected Topics in Applied Earth Observations and Remote Sensing, vol. 7, no. 8, pp. 3402-3415, 2014.

[8] P. R. Coppin and M. E. Bauer, "Digital change detection in forest ecosystems with remote sensing imagery," Remote Sensing Reviews, vol. 13, no. 3-4, pp. 207-234, 1996.

[9] N. A. Quarmby and J. L. Cushnie, "Monitoring urban land cover changes at the urban fringe from SPOT HRV imagery in southeast England," International Journal of Remote Sensing, vol. 10, no. 6, pp. 953-963, 1989.

[10] Y. Bazi, L. Bruzzone, and F. Melgani, "An unsupervised approach based on the generalized Gaussian model to automatic change detection in multitemporal SAR images," IEEE Transactions on Geoscience and Remote Sensing, vol. 43, no. 4, pp. 874-886, 2005.

[11] J. Inglacla and G. Mercier, "A new statistical similarity measure for change detection in multitemporal SAR images and its extension to multiscale change analysis," IEEE Transactions on Geoscience and Remote Sensing, vol. 45, no. 5, pp. 1432-1445, 2007.

[12] A. K. Ludeke, R. C. Maggio, and L. M. Reid, "An analysis of anthropogenic deforestation using logistic regression and GIS," Journal of Environmental Management, vol. 31, no. 3, pp. 247259, 1990.
[13] E. H. Wilson and S. A. Sader, "Detection of forest harvest type using multiple dates of landsat TM imagery," Remote Sensing of Environment, vol. 80, no. 3, pp. 385-396, 2002.

[14] M.-L. Nordberg and J. Evertson, "Vegetation index differencing and linear regression for change detection in a Swedish mountain range using Landsat TM and ETM+imagery," Land Degradation and Development, vol. 16, no. 2, pp. 139-149, 2005.

[15] J. S. Deng, K. Wang, Y. H. Deng, and G. J. Qi, "PCA-based land-use change detection and analysis using multitemporal and multisensor satellite data," International Journal of Remote Sensing, vol. 29, no. 16, pp. 4823-4838, 2008.

[16] N. Otsu, "A threshold selection method from gray-level histograms," Automatica, vol. 11, pp. 23-27, 1975.

[17] L. Li, X. Li, Y. Zhang, L. Wang, and G. Ying, "Change detection for high-resolution remote sensing imagery using objectoriented change vector analysis method," in Proceedings of the 36th International Geoscience and Remote Sensing Symposium, IGARSS '16, pp. 2873-2876, IEEE, Beijing, China, July 2016.

[18] K. G. Pillai and R. R. Vatsavai, "Multi-sensor remote sensing image change detection: an evaluation of similarity measures," in Proceedings of the 2013 13th International Conference on Data Mining Workshops, ICDMW '13, pp. 1053-1060, IEEE, Dallas, Tex, USA, December 2013.

[19] M. A. Hossain, X. Jia, and M. Pickering, "Subspace detection using a mutual information measure for hyperspectral image classification," IEEE Geoscience and Remote Sensing Letters, vol. 11, no. 2, pp. 424-428, 2014.

[20] J. Prendes, M. Chabert, F. Pascal, A. Giros, and J.-Y. Tourneret, "A new multivariate statistical model for change detection in images acquired by homogeneous and heterogeneous sensors," IEEE Transactions on Image Processing, vol. 24, no. 3, pp. 799812, 2015.

[21] S. Cui, G. Schwarz, and M. Datcu, "A benchmark evaluation of similarity measures for multitemporal SAR image change detection," IEEE Journal of Selected Topics in Applied Earth Observations and Remote Sensing, vol. 9, no. 3, pp. 1101-1118, 2016.

[22] A. A. Nielsen and J. S. Vestergaard, "Change detection in bitemporal data by canonical information analysis," in Proceedings of the 8th International Workshop on the Analysis of Multitemporal Remote Sensing Images, Multi-Temp '15, IEEE, Annecy, France, July 2015.

[23] L. An, M. Li, P. Zhang, Y. Wu, L. Jia, and W. Song, "Multicontextual mutual information data for SAR image change detection," IEEE Geoscience and Remote Sensing Letters, vol. 12, no. 9, 2015.

[24] E. Erten, A. Reigber, L. Ferro-Famil, and O. Hellwich, "A new coherent similarity measure for temporal multichannel scene characterization," IEEE Transactions on Geoscience and Remote Sensing, vol. 50, no. 7, pp. 2839-2851, 2012.

[25] T. M. Cover and J. A. Thomas, Elements of Information Theory, John Wiley \& Sons, Hoboken, NJ, USA, 2nd edition, 2012.

[26] S. Liu, L. Bruzzone, F. Bovolo, and P. Du, "Hierarchical unsupervised change detection in multitemporal hyperspectral images," IEEE Transactions on Geoscience and Remote Sensing, vol. 53, no. 1, pp. 244-260, 2015.

[27] M. İlsever and C. Ünsalan, Two-Dimensional Change Detection Methods, Springer Briefs in Computer Science, 2012. 


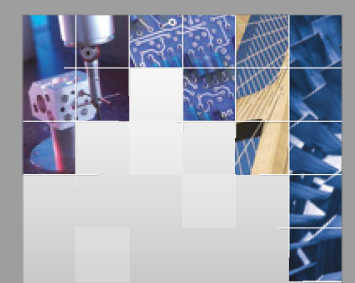

\section{Enfincering}
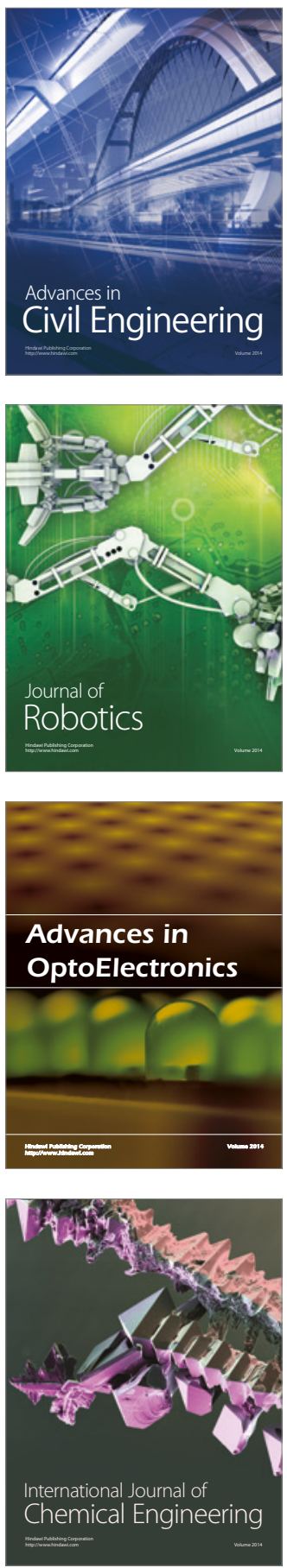

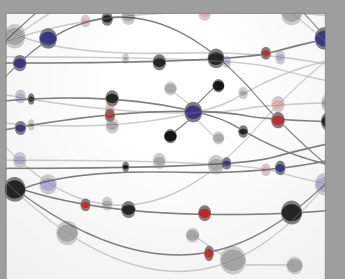

The Scientific World Journal

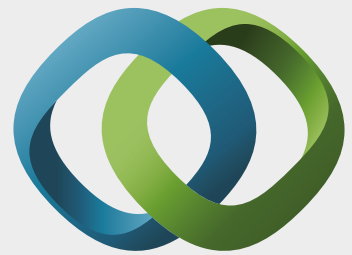

\section{Hindawi}

Submit your manuscripts at

https://www.hindawi.com
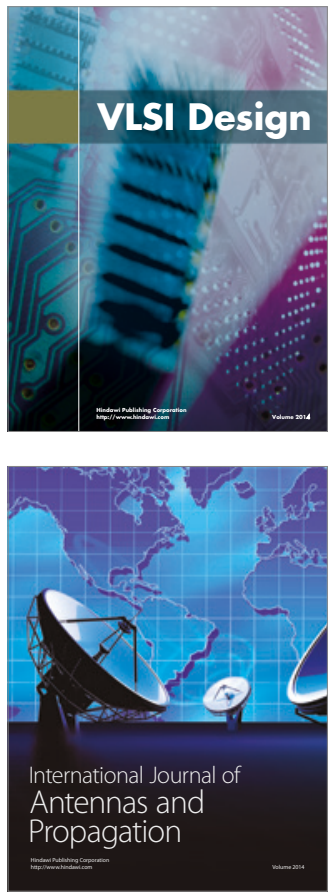

\section{Rotating}

Machinery
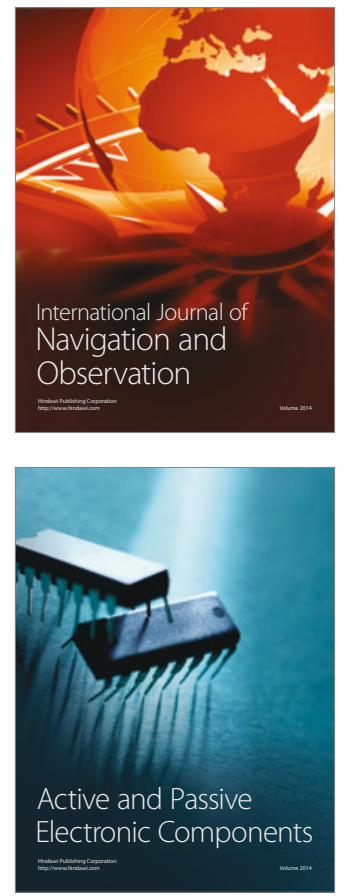
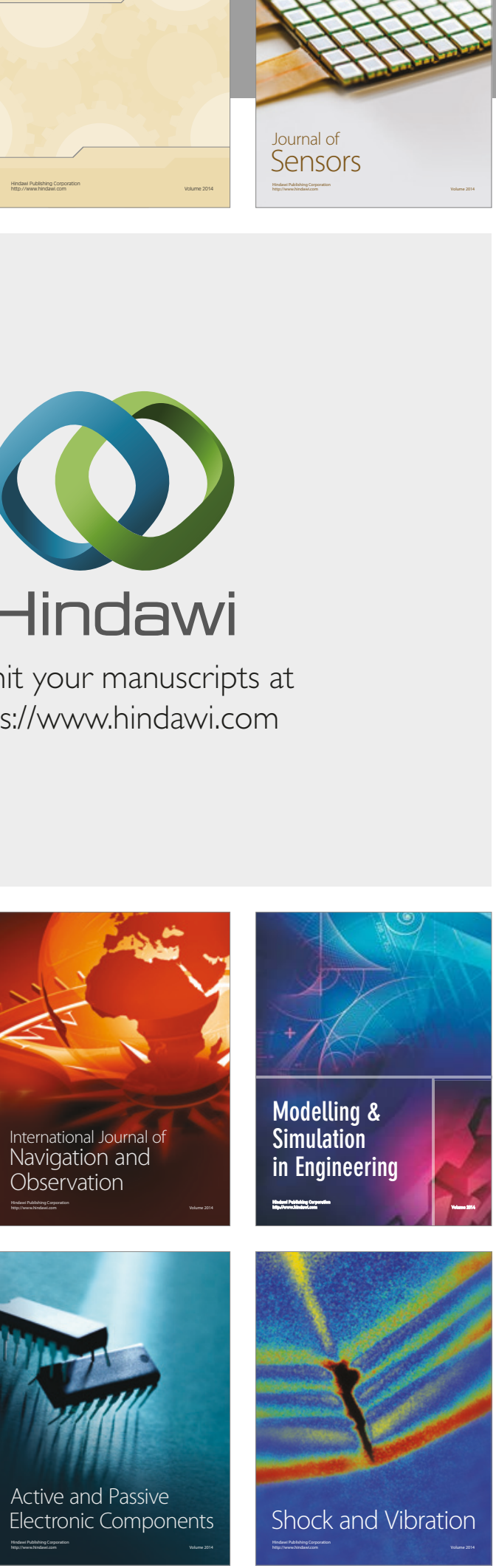
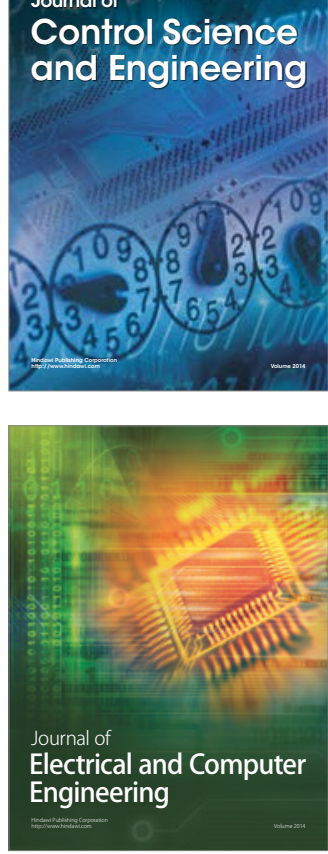

Distributed

Journal of

Control Science

and Engineering
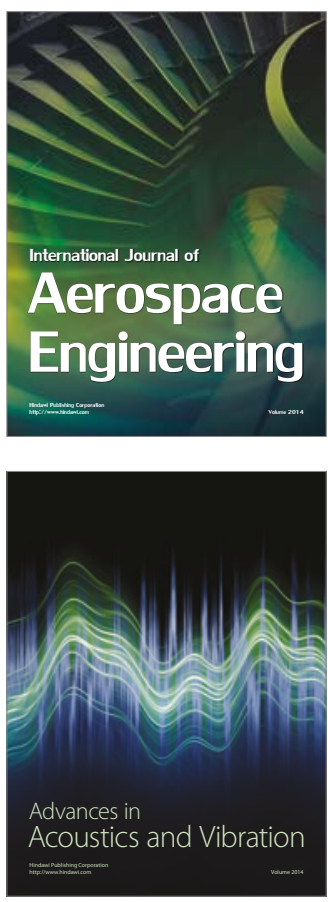

Sensor Networks 